\title{
USP18-deficiency in cervical carcinoma is crucial for the malignant behavior of tumor cells in an ERK signal-dependent manner
}

\author{
AONAN PAN ${ }^{1}$, YUE LI $^{2}$, JIAN GUAN ${ }^{3}$, PENGXIA ZHANG $^{4}$, CHUNBIN ZHANG $^{4}$, YUPENG HAN $^{5}$,

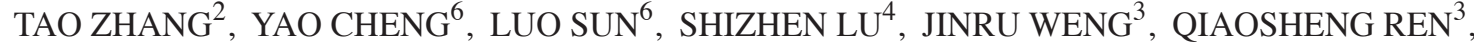 \\ SHENGJIE FAN ${ }^{7}$, WEIQUN WANG ${ }^{8}$ and JINGTAO WANG ${ }^{9}$
}

\begin{abstract}
${ }^{1}$ Department of Clinical Medicine, The Affiliated Second Hospital, Harbin Medical University, Harbin, Heilongjiang 150081;
${ }^{2}$ Departments of Immunology and Etiology, Basic Medical College, Jiamusi University, Jiamusi, Heilongjiang 154007;

${ }^{3}$ Department of Maxillofacial Surgery, Stomatological College, Jiamusi University, Jiamusi, Heilongjiang 154002;

${ }^{4}$ Department of Biochemistry and Cell and Molecular Biology, Basic Medical College, Jiamusi University, Jiamusi,

Heilongjiang 154007; Departments of ${ }^{5}$ Gastroenterology and ${ }^{6}$ Clinical Laboratory, The First Affiliated Hospital,

Jiamusi University, Jiamusi, Heilongjiang 154002; ${ }^{7}$ Department of Rehabilitation Medicine, Rehabilitation Medical College,

Jiamusi University, Jiamusi, Heilongjiang 154007; Departments of ${ }^{8}$ Physiology and ${ }^{9}$ Human Anatomy,

Basic Medical College, Jiamusi University, Jiamusi, Heilongjiang 154007, P.R. China
\end{abstract}

Received November 14, 2020; Accepted February 25, 2021

DOI: $10.3892 / \mathrm{ol} .2021 .12682$

\begin{abstract}
Ubiquitin-specific peptidase (USP)18 belongs to the USP family, and is involved in cleaving and removing ubiquitin or ubiquitin-like molecules from their target molecules. Recently, increasing evidence has suggested that USP18 is constitutively expressed in different types of human tumors, and ectopic expression or downregulation of USP18 expression may contribute to tumorigenesis. However, the role of USP18 in uterine cervical cancer (UCC) remains unclear. Thus, the present study aimed to investigate USP18 expression in a human tissue microarray constructed using UCC and non-cancer cervical tissues, and to determine the potential role and molecular mechanism by which USP 18 is implicated in the tumor biology of human UCC HeLa cells. Microarray analysis demonstrated that USP18 protein expression was downregulated in tumor tissues compared with in normal tissues. In addition, in vitro analysis revealed that USP18-knockdown markedly promoted the
\end{abstract}

Correspondence to: Professor Weiqun Wang, Department of Physiology, Basic Medical College, Jiamusi University, 258 Xuefu Street, Jiamusi, Heilongjiang 154007, P.R. China

E-mail: wangweiqun1974@163.com

Professor Jingtao Wang, Department of Human Anatomy, Basic Medical College, Jiamusi University, 258 Xuefu Street, Jiamusi, Heilongjiang 154007, P.R. China

E-mail:wjingtao@jmsu.edu.cn

Key words: uterine cervical carcinoma, ubiquitin-specific peptidase 18, malignant behavior, ERK proliferation, colony formation, migration and aggressiveness of HeLa cells. Mechanistic analysis demonstrated that USP18-knockdown increased the levels of Bcl-2, STAT3 and phosphorylated-ERK in HeLa cells. Notably, USP18 silencing-induced malignant phenotypes were interrupted following exogenous administration of the ERK1/2 inhibitor PD98059. Overall, the results of the present study suggested that USP18 may be a potent inhibitor involved in UCC tumor-associated biological behaviors, which are associated with the ERK signaling pathway.

\section{Introduction}

Uterine cervical carcinoma (UCC) is one of the most common types of malignant cancer in women, representing the fourth most frequent malignancy worldwide (1). In 2018, $\sim 570,000$ new cases of UCC were identified, and up to 311,000 associated deaths were recorded worldwide (2). Epidemiological data have demonstrated that the incidence and mortality rates of UCC vary across different regions, with more cases in Sub-Saharan Africa and South-Eastern Asia, and less cases in North America, Australia, New Zealand and Western Asia (2). Although it has been reported that UCC incidence and mortality rates have decreased in several regions of the world over the last few decades (3), UCC remains a serious health issue in China, with an estimated 106,430 new cases and 47,739 mortalities in 2018 (3).

Ubiquitin-specific peptidase (USP)18, also known as ubiquitin-specific protease 43 (UBP43), is a member of the USP family and is involved in deubiquitinating activity, thereby resulting in stabilization of substrates (4). It is well known that USPs are the largest sub-family of deubiquitinase enzymes, and exert biological roles through their cysteine 
endopeptidase activity (5). The USP family consists of $>100$ members, which predominantly differ in amino acid sequence and protein size, but are characterized by several highly homologous sequences around the essential domains important for their catalytic activity $(6,7)$. USP18 was originally identified from acute myelogenous leukemia 1- RUNX1 partner transcriptional co-repressor 1 knock-in mice and was characterized by Liu et al (8). Previous studies have demonstrated that USP18 expression is present in multiple types of tissues, including liver, lung, spleen, thymus, bone marrow and adipose tissues $(8,9)$, and is also expressed in different types of cells, such as macrophages, lymphocytes and hematopoietic cells $(10,11)$. The function of USP18 has predominantly been associated with the regulation of cell proliferation, cell differentiation, stress, inflammatory reaction and immune response (9-11). Additionally, it has been suggested that USP18 serves a vital role in regulating T-cell activation and $\mathrm{T}$ helper 17 cell differentiation through its ability to remove the transforming growth factor $\beta$-activated kinase 1-TAK1-binding protein 1 complex (12).

Several studies have suggested that USP18 may be involved in tumor biology $(10,13)$. USP18 is overexpressed in several types of human cancer including glioblastoma, hepatocellular carcinoma, bladder cancer and breast cancer, and its high expression is associated with a poor prognosis in patients with glioblastoma and bladder cancer (14-17). Furthermore, some studies have demonstrated that USP18 is important for the malignant behaviors of tumor cells, including cellular proliferation, migration, apoptosis and epithelial-to-mesenchymal transition (EMT) (14,17-19). Conversely, it has been demonstrated that USP18 may suppress tumorigenesis due to its involvement in the antitumor immune response (20).

Although the biological functions and clinical presentation of USP18 have been well characterized in several types of human cancer including glioblastoma, breast cancer and melanoma $(14,17,19,20)$, its underlying molecular mechanisms in UCC remain unclear. Thus, the present study aimed to investigate USP18 expression in a cervix tissue microarray, and determine its potential role and molecular mechanism in UCC malignant phenotypes.

\section{Materials and methods}

Cell line, cell culture and cell transfection. Human UCC HeLa cells were purchased from the American Type Culture Collection and maintained in RPMI-1640 medium supplemented with $10 \%$ fetal bovine serum, $100 \mathrm{U} / \mathrm{ml}$ penicillin and $100 \mathrm{mg} / \mathrm{ml}$ streptomycin (all Gibco; Thermo Fisher Scientific, Inc.) at $37^{\circ} \mathrm{C}$ with $5 \% \mathrm{CO}_{2}$ in a humidified incubator.

To generate USP18-deficient HeLa cells, an RNA interference silencing strategy was used to design and construct a lentivirus vector carrying small interfering (si)RNA sequences targeting USP18 by GeneCopoeia, Inc. The lentiviruses containing USP18-siRNA vector and scrambled negative control vector (mock) were synthesized by GeneCopoeia, Inc (cat. nos. HSH117922-LVRU6GP-c and CSHCTR0013-LVRU6GP, respectively). The sequences targeting USP18 were 5'-CCA ACATTA ATTCCATATGAA-3', and the scrambled sequences of 5'-ACGCGTATTCGTTTACTGT-3' were used as negative control. Following infection with the vector-carrying lentiviruses, according to the manufacturer's protocol, cells were subsequently treated with $2 \mathrm{ng} / \mathrm{ml}$ puromycin (Gibco; Thermo Fisher Scientific, Inc.) and transfection efficiency was observed under a fluorescence microscope (magnification, x200). Western blot analysis was subsequently performed to detect USP18 protein expression.

Immunohistochemistry (IHC). A commercial cervix tissue microarray containing 15 squamous cell carcinoma tissues, five adenosquamous carcinoma tissues, 20 adenocarcinoma tissues, 20 cervical intraepithelial lesions tissues, 14 cervicitis tissues, four unpaired para-cancerous tissues and two cervical canal tissues (cat. no. F801301; Bioaitech Co., Ltd.) was used to detect USP18 expression. The characteristics of the tissues are listed in Table I.

The microarray was processed routinely via deparaffinization, rehydration, endogenous peroxidase quenching and antigen retrieval, and subsequently blocked with $20 \%$ normal goat serum (Wuhan Boster Biological Technology, Ltd.) for $30 \mathrm{~min}$ at $37^{\circ} \mathrm{C}$ to remove the background from staining. The microarray was incubated with a mouse antibody against human USP18 (1:50; cat. no. sc-374064; Santa Cruz Biotechnology, Inc.) overnight at $4^{\circ} \mathrm{C}$. Following the primary antibody incubation, the microarray was incubated for $35 \mathrm{~min}$ at $37^{\circ} \mathrm{C}$ using the SABC kit (cat. no. SA1021; Wuhan Boster Biological Technology, Ltd.). DAB and hematoxylin were used for visualization and nuclear counterstaining at room temperature for 30 and $5 \mathrm{sec}$, respectively. The USP18 protein expression profiles were estimated based on the staining intensity and the percentage of positive cells using a fluorescence microscope (magnification, x200). The staining intensity was ranked as follows: 1 , weak; 2 , moderate; 3 , intensive; and 4, super intensive. The percentage of positive cells was scored as follows: $1,<25 \% ; 2,26-50 \% ; 3,51-75 \%$; and $4,>75 \%$. The total score for each sample was the sum of the two parameters, as previously described (21).

Cell proliferation assay. The Cell Counting Kit- 8 (CCK-8; Wuhan Boster Biological Technology, Ltd.) assay was performed to assess the proliferation of $\mathrm{HeLa}$ cells following USP18-knockdown. Briefly, mock- and USP18-siRNA-transfected HeLa cells were seeded into 96 -well plates at a density of $1.5 \times 10^{3}$ cells/well in a final volume of $100 \mu \mathrm{l}$ RPMI-1640 medium supplemented with or without $80 \mu \mathrm{M}$ of the ERK1/2 blocker PD98059 (Sigma-Aldrich; Merck KGaA). Following incubation at $37^{\circ} \mathrm{C}$ for 24,48 or $72 \mathrm{~h}$, $10 \mu \mathrm{l}$ CCK-8 reagent was added to each well and incubated at $37^{\circ} \mathrm{C}$ for $1 \mathrm{~h}$. Cell proliferation was subsequently analyzed at a wavelength of $450 \mathrm{~nm}$, using a microplate reader (Omega Bio-Tek, Inc.). The inhibitory role of PD98059 on the proliferation of HeLa cells was estimated as follows: Inhibition rate (\%) $=($ treatment with $0 \mu \mathrm{M}$ PD98059-treatment with $80 \mu \mathrm{M}$ PD98059)/treatment with $0 \mu \mathrm{M}$ PD98059 x100.

Clonogenicabilityassay.Mock-andUSP18-siRNA-transfected HeLa cells were seeded into 24-well plates at a density of $5 \times 10^{2}$ cells/well in $500 \mu \mathrm{l}$ RPMI-1640 medium supplemented with $10 \%$ fetal bovine serum. Cells were cultured at $37^{\circ} \mathrm{C}$ and the medium was replaced every 2-3 days. Following incubation for 10 days, the medium was removed and cells were stained with $0.01 \%$ crystal violet for $15 \mathrm{~min}$ at room temperature. 
A minimum of $3 \mathrm{~mm}$ diameter or more was considered as a colony. Images of cell colonies were captured using an imaging system (Tanon Science \& Technology Co., Ltd.) and counted under a fluorescence microscope (magnification, x50).

Cell migration assay. The migratory ability of HeLa cells was assessed using 24-well Transwell chambers with polycarbonate filter of $8-\mu \mathrm{m}$ pore size. A total of $2 \times 10^{4}$ mock- and USP18-siRNA-transfected HeLa cells were plated in the upper chambers of Transwell plates in $100 \mu \mathrm{l}$ serum-free RPMI-1640 medium. A total of $600 \mu \mathrm{l}$ RPMI-1640 medium containing $10 \%$ FBS (Gibco; Thermo Fisher Scientific, Inc.) supplemented with or without $80 \mu \mathrm{M}$ of the ERK1/2 blocker PD98059 was plated in the lower chambers. Following incubation for $24 \mathrm{~h}$ at $37^{\circ} \mathrm{C}$, cells in the upper chambers were removed using a cotton swab, while the migratory cells were fixed and stained using a solution of crystal violet in ethanol for $15 \mathrm{~min}$ at room temperature. Stained cells were counted using a fluorescence microscope (magnification, x200). The inhibitory role of PD98059 on the migration of HeLa cells was estimated as follows: Inhibition rate $(\%)=($ treatment with $0 \mu \mathrm{M}$ PD98059-treatment with $80 \mu \mathrm{M}$ PD98059)/treatment with $0 \mu \mathrm{M}$ PD98059 x100.

Wound healing assay. Mock- and USP18-siRNA-transfected HeLa cells were seeded into 6-well plates at a density of $2 \times 10^{6}$ cells/well in $1 \mathrm{ml}$ RPMI-1640 medium supplemented with $10 \%$ fetal bovine serum. After incubation until confluent, the culture medium was replaced with serum-free medium, and a cell-free wound zone was created by scraping the monolayer with a sterile pipette tip. The images of the wounds were captured and the numbers of migrating cells were counted at 0 and $24 \mathrm{~h}$ after wounding using a fluorescence microscope by eye (magnification, x100).

Western blotting. Untransfected parental, mock- and USP18-siRNA-transfected HeLa cells were harvested and lysed using a commercial RIPA buffer kit (cat. no. P0013C; Beyotime Institute of Biotechnology) supplemented with phenylmethylsulfonyl fluoride. Protein concentrations were determined using the bicinchoninic acid protein assay kit (cat. no. P0010; Beyotime Institute of Biotechnology) and $40 \mu \mathrm{g}$ protein/lane was subjected to $12 \%$ SDS-PAGE. The separated proteins were subsequently transferred onto polyvinylidene difluoride membranes and blocked with $5 \%$ skimmed milk in PBS for $60 \mathrm{~min}$ at $37^{\circ} \mathrm{C}$. The membranes were incubated with primary antibodies against USP18 (1:1,000; cat. no. DF7968; Affinity Biosciences), Bcl-2 (1:1,000; cat. no. AF6139; Affinity Biosciences), STAT3 (1:1,000; cat. no. CY5165; Shanghai Abways Biotechnology Co., Ltd.), ERK (1:1,000; cat. no. CY5487; Shanghai Abways Biotechnology Co., Ltd.), phosphorylated (p)-ERK (1:1,000; cat. no. CY5277; Shanghai Abways Biotechnology Co., Ltd.) and $\beta$-actin (1:5,000; cat. no. AB0011; Shanghai Abways Biotechnology Co., Ltd.) overnight at $4^{\circ} \mathrm{C}$. Following the primary antibody incubation, membranes were incubated with HRP-coupled goat anti-rabbit and anti-mouse secondary antibodies (1:10,000; cat. nos. BA1054 and BA1050, respectively; Wuhan Boster Biological Technology, Ltd.). Protein blots were visualized using ECL reagent (Thermo Fisher Scientific, Inc.). The protein expression levels of USP18, Bcl-2 and STAT3 were normalized to $\beta$-actin, whereas $\mathrm{p}$-ERK expression was
Table I. Patient characteristics of tissues in cervix tissue microarray.

\begin{tabular}{lcc}
\hline Characteristics & $\begin{array}{c}\text { Cancer tissues, } \\
\mathrm{n}=40\end{array}$ & $\begin{array}{c}\text { Non-cancer tissues, } \\
\mathrm{n}=40\end{array}$ \\
\hline Age, years & & $32-66$ \\
Range & $26-63$ & 48.20 \\
Median & 48.68 & \\
Stage, $\mathrm{n}$ & & \\
I & 29 & \\
II & 9 \\
III & 2 \\
Grade, $\mathrm{n}$ & \\
1 & 10 \\
2 & 15 \\
3 & 10 \\
Lymph node & \\
metastasis, $\mathrm{n}$ & \\
Yes & 4 \\
No & 34 \\
\hline
\end{tabular}

normalized to total ERK expression using Image J software (version $1.45 \mathrm{~s}$; National Institutes of Health).

Statistical analysis. Statistical analysis was performed using SPSS 22.0 software (IBM Corp.). All experiments were performed in triplicate and data are presented as the mean \pm SD. Comparisons between two groups were analyzed using unpaired Student's t-test (if the variance was homogeneous) or Cochran and Cox separate variance estimation t-test (if the variance was not homogeneous). Comparisons among multiple groups were analyzed using one-way ANOVA followed by Student-Newman-Keuls post-hoc test. $\mathrm{P}<0.05$ was considered to indicate a statistically significant difference.

\section{Results}

USP18 expression is downregulated in UCC tissues. To evaluate the clinical significance of USP18, USP18 expression was determined via IHC analysis in a commercial cervix tissue microarray (Fig. 1). The final score demonstrated that USP18 was expressed at significantly lower levels in UCC tissues compared with in normal tissues (Table II). However, there was no significant difference in USP18 expression between different stages, grades, age and lymph node metastasis (Table II).

USP18 is expressed in HeLa cells. To assess the potential involvement of USP18 in the malignant phenotypes of HeLa cells, USP18 protein expression was detected. Western blot analysis confirmed that USP18 protein was expressed in untransfected parental HeLa cells (Fig. 2A). Following transfection with lentiviruses, fluorescent imaging demonstrated that siRNA- and mock-transfected cells expressed high-intensity screening fluorescence, suggesting that the cells were effectively infected by lentiviruses (Fig. 2B). Subsequent western blotting showed that USP18 protein expression was significantly decreased in siRNA-transfected HeLa 


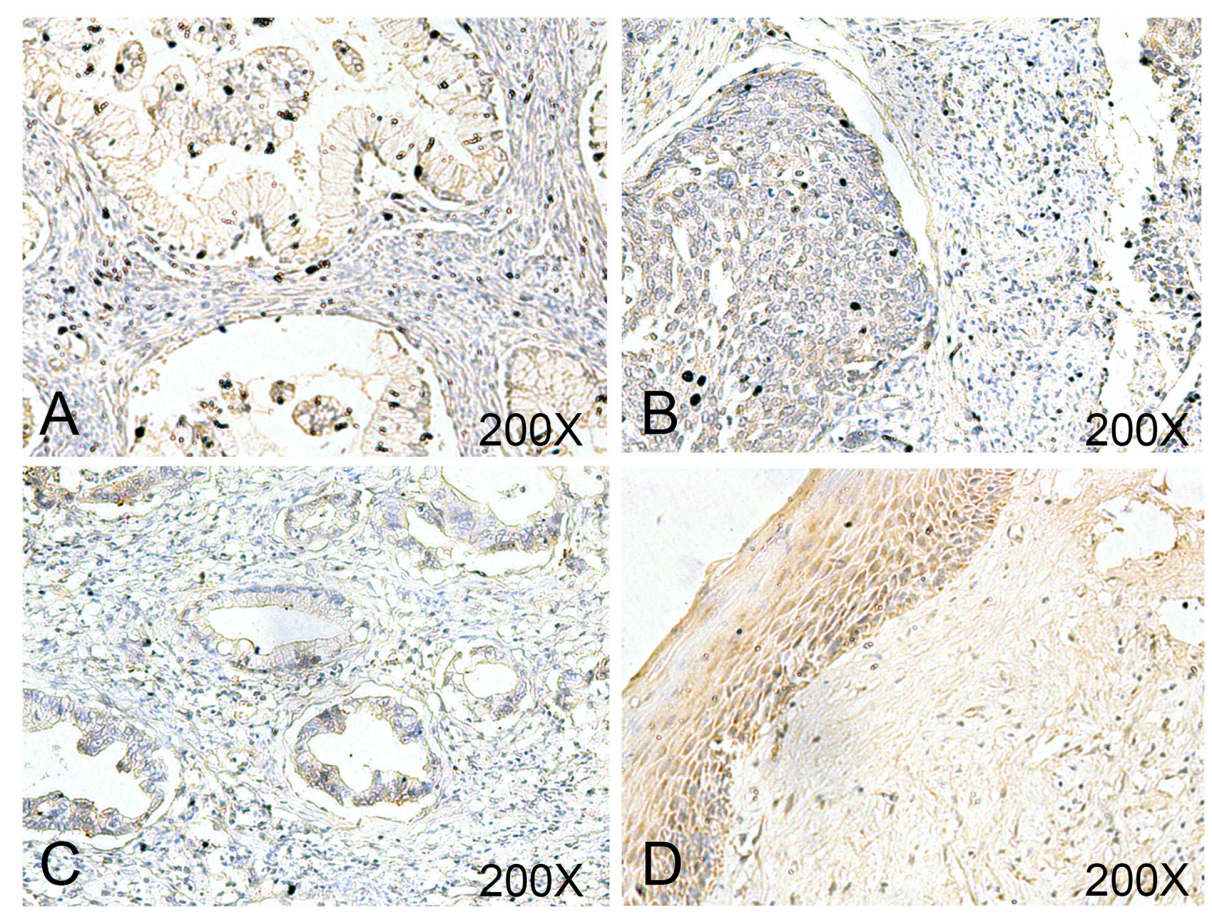

Figure 1. Downregulated USP18 expression in tumor tissues of a cervix tissue microarray. UCC tissues with grade (A) 1 and (B) 2 exhibited moderate staining, and grade (C) 3 exhibited weak staining of USP18. (D) Non-cancer tissues exhibited super intensive staining of USP18. Magnification, x200. USP18, ubiquitin-specific peptidase 18.

Table II. Ubiquitin-specific peptidase 18 expression in cervix tissue microarray (mean $\pm S$ ).

\begin{tabular}{llcl}
\hline Characteristics & USP18 level & $\mathrm{t}$ & P-value \\
\hline $\begin{array}{l}\text { Tissues } \\
\text { Non-cancer }\end{array}$ & $5.25 \pm 1.532$ & 2.1750 & 0.0327 \\
Cancer & $4.50 \pm 1.553$ & & \\
Stage & & & \\
I & $4.62 \pm 1.568$ & 0.7945 & 0.4318 \\
II-III & $4.18 \pm 1.537$ & & \\
Grade & & & \\
1 & $5.00 \pm 1.155$ & 1.0299 & 0.3105 \\
$2-3$ & $4.40 \pm 1.683$ & & \\
Age, years & & & \\
$\quad \leq 50$ & $4.35 \pm 1.522$ & 0.8511 & 0.4001 \\
$\geq 50$ & $4.79 \pm 1.626$ & & \\
Lymph node & & & \\
metastasis & & & \\
No & $4.62 \pm 1.615$ & 1.0452 & \\
Yes & $3.75 \pm 0.957$ & & \\
\hline
\end{tabular}

cells compared with untransfected parental and mock-transfected HeLa cells (Fig. 2A and C), which indicated that USP18-deficient HeLa cells were successfully established.

Downregulation of USP18 promotes the proliferation, colony formation, migration and aggressiveness of HeLa cells. The CCK-8 assay was performed to assess the effect of USP18
A

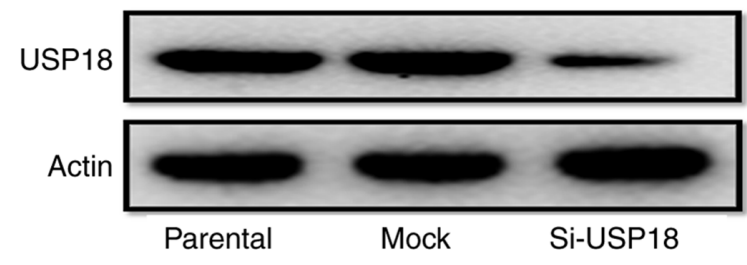

B
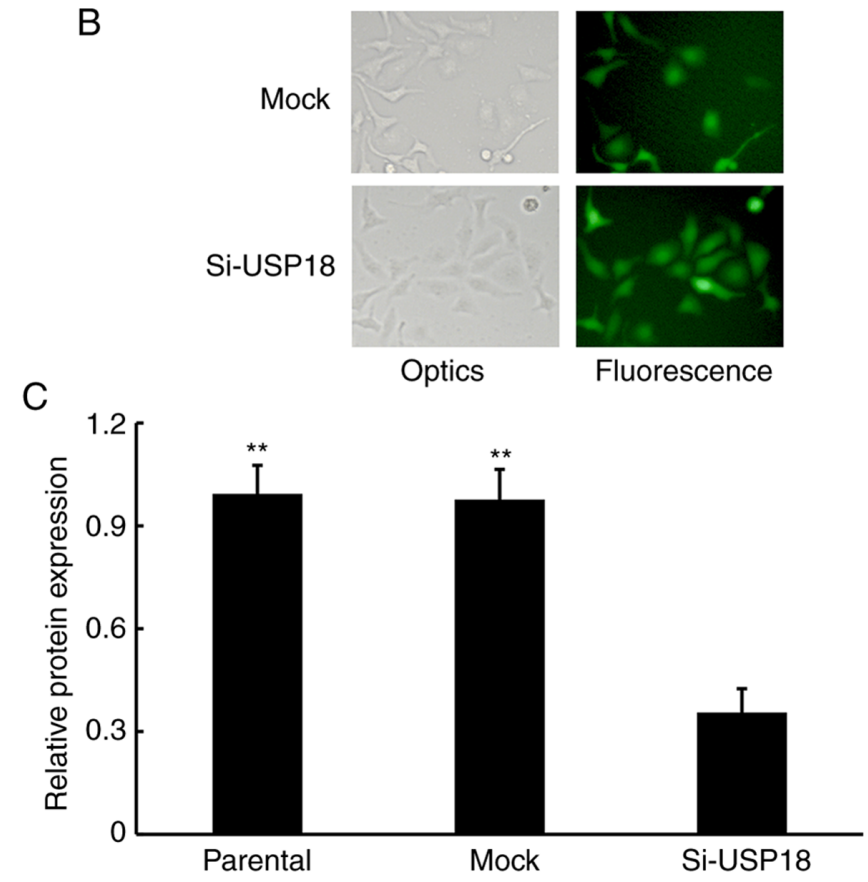

Figure 2. USP18 expression in HeLa cells. (A) Representative images of USP18 protein expression from western blot assay. (B) Fluorescence images of mock- and USP18-siRNA-transfected HeLa cells. Magnification, x200. (C) Statistical analysis of USP18 protein expression. ${ }^{* *} \mathrm{P}<0.01$ vs. si-USP18. USP18, ubiquitin-specific peptidase 18; siRNA, small interfering RNA. 
A

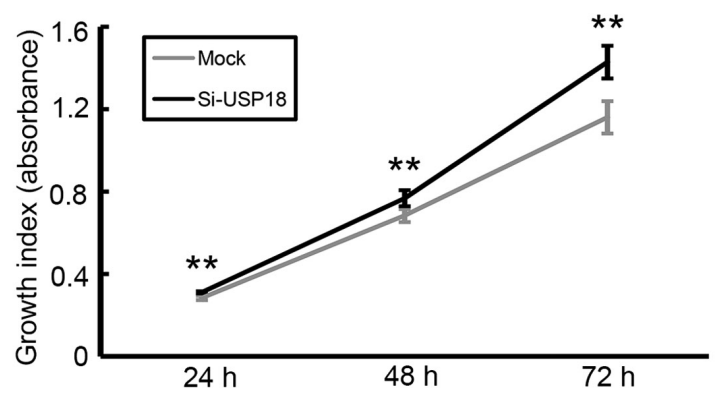

B
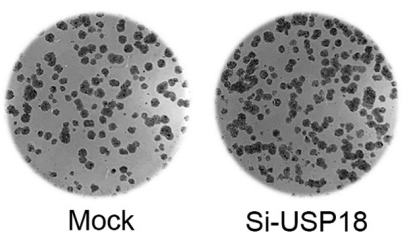

D

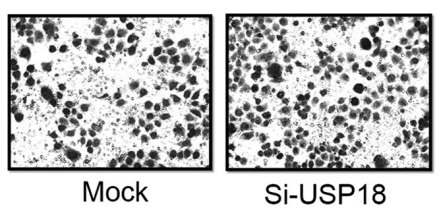

$\mathrm{F}$

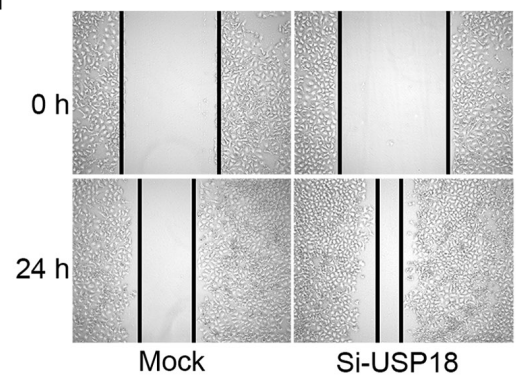

C

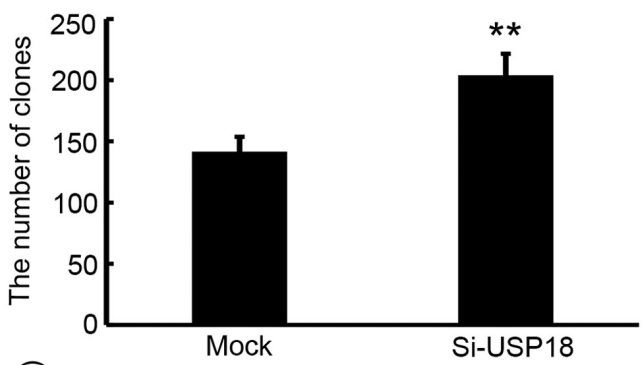

E

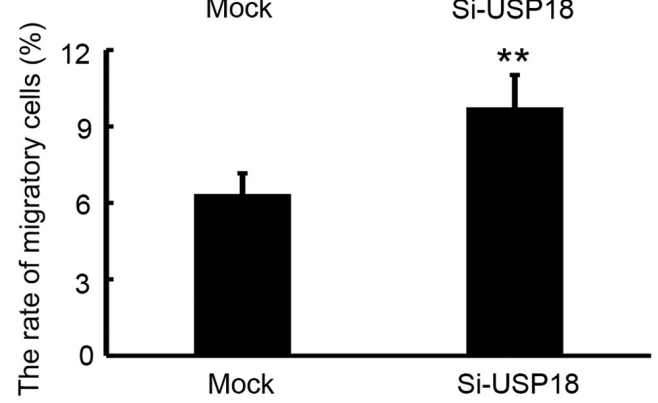

G

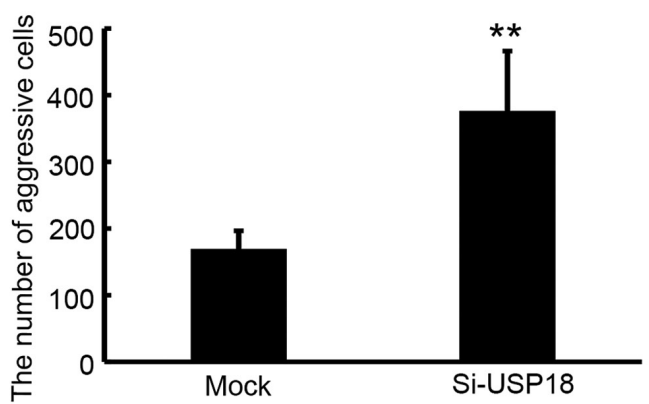

Figure 3. Roles of USP18-deficiency in HeLa cell malignant behaviors in vitro. (A) Proliferation analysis from mock- and USP18-siRNA-transfected HeLa cells using Cell Counting Kit-8 reagent. (B) Representative images of clonogenic analysis from mock- and USP18-siRNA-transfected HeLa cells. (C) Statistical analysis of colony formation ability. (D) Representative images of Transwell analysis from mock- and USP18-siRNA-transfected HeLa cells. Magnification, x200. (E) Statistical analysis of cell migratory capacity. (F) Representative images of wound healing assay from mock- and USP18-siRNA-transfected HeLa cells. Magnification, x100. (G) Statistical analysis of cell aggressiveness capacity. ${ }^{* *} \mathrm{P}<0.01$ vs. mock. USP18, ubiquitin-specific peptidase 18 ; siRNA, small interfering RNA.

on the malignant behavior of HeLa cells. Following incubation for 24,48 and $72 \mathrm{~h}$, the results demonstrated that the proliferative ability of siRNA-transfected HeLa cells was significantly increased compared with mock-transfected HeLa cells (Fig. 3A). The results of the colony formation, migration and wound healing assays demonstrated that silencing of USP18 in HeLa cells significantly increased their colony forming (Fig. 3B and C), migratory (Fig. 3D and E) and aggressive abilities (Fig. 3F and G).

USP18-knockdown alters ERK-signaling pathway-specific protein expression. The potential molecular mechanism by which USP18 regulates the malignant process of HeLa cells was assessed via western blotting. The results demonstrated that the protein expression levels of Bcl-2, STAT3 (Fig. 4A and B) and p-ERK (Fig. 4C and D) were significantly increased following USP18-knockdown in HeLa cells, suggesting the involvement of ERK signaling in the malignant phenotypes of UCC cells.

USP18 silencing-induced malignant behaviors of HeLa cells depend on ERK signaling. To further clarify whether the roles of USP18 silencing on HeLa cells were mediated by ERK signaling, the ERK1/2 blocker PD98059 was used. The results demonstrated that there were no significant differences in cell proliferation at 24 or $48 \mathrm{~h}$ (Fig. 5A) and cell migration at $24 \mathrm{~h}$ (Fig. 5C and D) between siRNA- and mock-transfected cells following treatment with PD98059. Although the proliferative rate of siRNA-transfected cells was significantly higher 


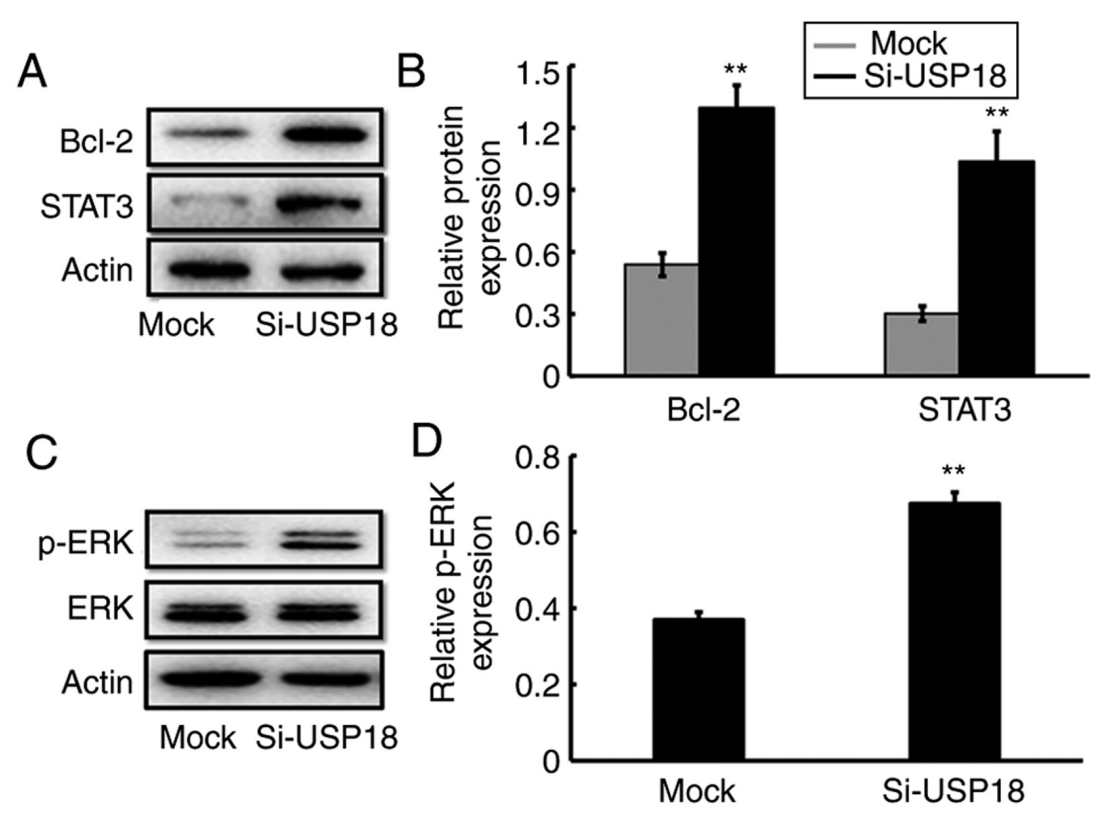

Figure 4. Effects of USP18-deficiency on regulating ERK-associated gene expression levels in HeLa cells. (A) Representative western blotting images of Bcl-2 and STAT3 from mock- and USP18-siRNA-transfected HeLa cells. (B) Quantification of Bcl-2 and STAT3 protein expression. (C) Representative western blotting images of ERK and p-ERK form mock- and USP18-siRNA-transfected HeLa cells. (D) Quantification of p-ERK levels. ${ }^{* *} \mathrm{P}<0.01$ vs. mock. USP18, ubiquitin-specific peptidase 18; siRNA, small interfering RNA; p, phosphorylated.

A

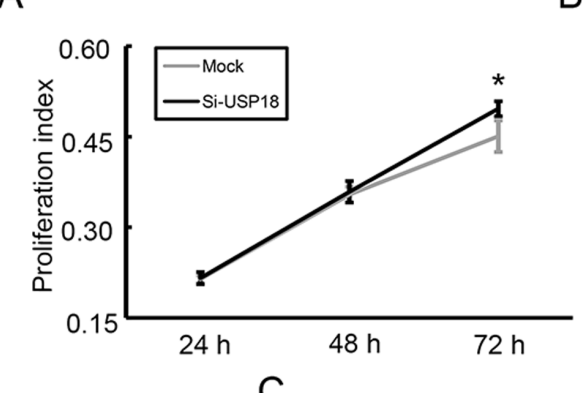

C

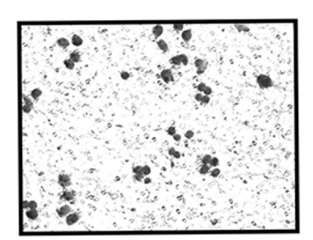

D

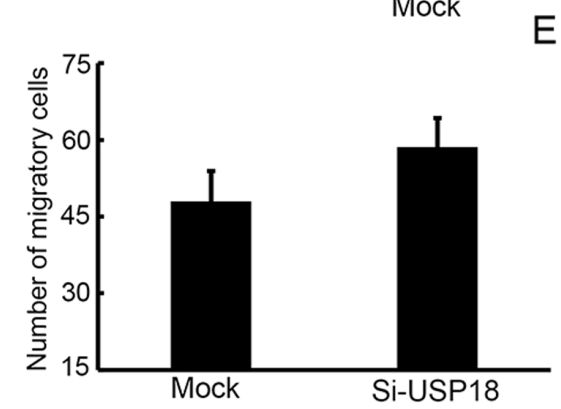

$\mathrm{B}$
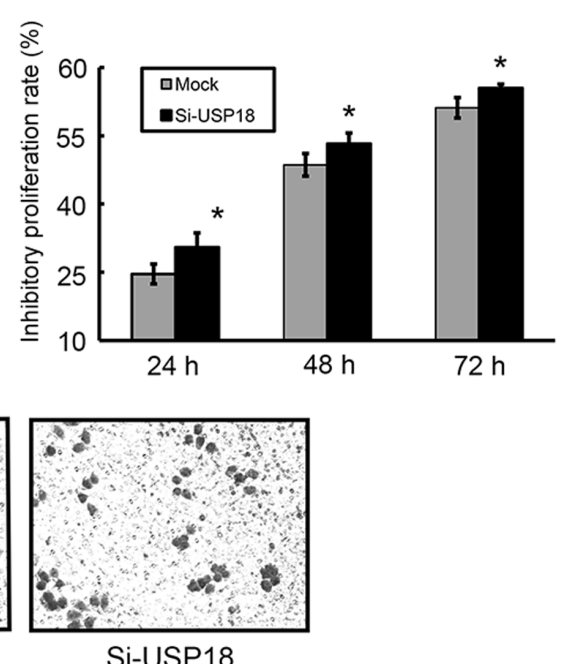

E

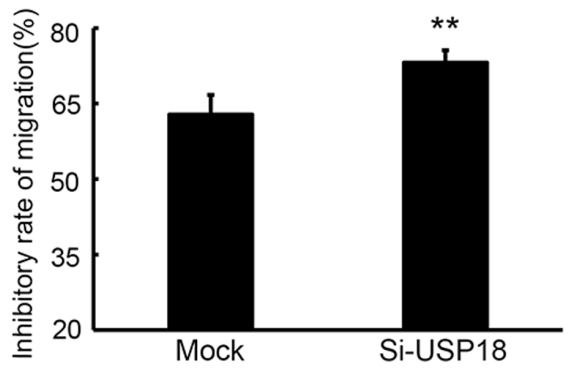

Figure 5. Involvement of ERK signaling in USP18-deficient-mediated malignant behaviors in HeLa cells. (A) Proliferation analysis after mock- and USP18-siRNA-transfected HeLa cells treatment with PD98059. (B) Proliferation inhibitory activity analysis after mock- and USP18-siRNA-transfected HeLa cells treatment with PD98059. (C) Representative images of Transwell analysis from mock- and USP18-siRNA-transfected HeLa cells treatment with PD98059. Magnification, x200. (D) Statistical analysis of cell migratory capacity. (E) Migration inhibitory activity analysis after mock- and USP18-siRNA-transfected HeLa cells treatment with PD98059. " $\mathrm{P}<0.05$ and $^{* * *} \mathrm{P}<0.01$ vs. mock. USP18, ubiquitin-specific peptidase 18; siRNA, small interfering RNA.

compared with that of mock-transfected cells after $72 \mathrm{~h}$ of exposure to PD98059 ( $\mathrm{P}<0.05$; Fig. 5A), the difference between the two groups was slightly lower compared with that between the two groups treated without PD98059 (Fig. 3A). Following 
treatment with PD98059, the inhibition rates exerted by PD98059 on the proliferation (Fig. 5B) and migration (Fig. 5E) of siRNA-transfected HeLa cells were significantly higher compared with those in mock-transfected HeLa cells. Overall, the present results suggested that USP18 silencing-induced malignant responses in HeLa cells depend on the activation of the ERK signaling pathway.

\section{Discussion}

USP18 is a major isopeptidase, which was initially identified based on its role to efficiently deconjugate interferon-stimulated gene 15 (ISG15), a two-domain ubiquitin-like protein, from ISGylation (9). In addition to ISG15, USP18 is highly induced by type I and III interferons, and it has been proposed that USP18 is a vital blocker of the type I interferons signaling pathway (22). Furthermore, a study demonstrated that type III interferons may induce USP18 production (23). In the presence of USP18, type III interferons acquire higher properties to weaken type I interferons-mediated actions by repressing JAK-STAT signaling (23).

Increasing evidence has suggested that USP18 is implicated in a variety of physiological and pathological processes in different tissues and cells, including cell development, viral infection, viral replication and antibacterial response $(10,24,25)$. However, a vast expansion in the understanding of USP18 expression and its association with tumor biology has occurred. It has been reported that USP18 is frequently overexpressed in different types of cancer, including breast cancer, bladder cancer and hepatocellular carcinoma, and its overexpression is positively associated with several pathological tumor characteristics (15-17). For example, a recent study indicated that USP18 methylation is predominantly downregulated, whereas its expression is upregulated in breast cancer, which is positively associated with increasing TNM stage, worse disease-free survival rate and HER2 ${ }^{+}$patients, but negatively associated with apoptosis (17). Accordingly, it has been suggested that USP18 may be used as a predictive marker for poor prognosis in muscle invasive bladder cancer, since high USP18 expression is a significant risk factor for cancer-specific death, and decreased USP18 expression is markedly associated with longer cancer-specific survival (16). USP18 has also been the focus of investigations evaluating its functions in tumorigenesis. USP18 silencing in a mouse model for breast cancer exhibited a significant decrease in tumor growth, and USP18-deficiency in breast cancer MCF-7 cells in vitro triggered an increase in the induction of apoptosis $(18,19)$. In addition, downregulation of USP18 expression in glioblastoma cells may protect against tumor cell invasion and migration by repressing EMT (14), an essential event for cancer metastasis, by which tumor cells obtain increased motility and invasiveness. Gain-of-function assays in vitro have demonstrated that overexpression of USP18 has an important role in regulating tumor progression due to its contribution in enhancing breast cancer tumor cell proliferation, colony formation and cell cycle progression (17). However, studies have also revealed that USP18 may exert an opposing role in the control of cancer development. For example, ectopic USP18 expression in B16 melanoma cancer cells may suppress tumorigenesis, restraining cancer cell-mediated inhibition of T-cell proliferation and activation, thus facilitating cancer cells to specific immune responses (20). In human leiomyosarcoma, downregulation of USP18 is associated with a poor clinical outcome, and USP18-deficient mice exhibited an enhanced ability to develop these sarcomas (26).

Although a recent study demonstrated that USP18 is a critical regulator for the tumorigenicity of cervical cancer CaSki and SiHa cells (27), the results of the present study demonstrated that USP18 expression was downregulated in UCC compared with in normal tissues. USP18 expression was knocked down in HeLa cells, and the malignant behaviors in cells, including proliferation, colony formation and migration, were enhanced. Mechanistically, p-ERK expression was significantly upregulated following USP18-knockdown in HeLa cells. Previous studies have reported that several signaling pathways, such as the PTEN/AKT (28), AKT/Skp2 (16) and JAK/STAT (29) signaling pathways, are implicated in USP18 associated-biological roles. To the best of our knowledge, the present study was the first to illustrate an involvement of ERK signaling in the function of USP18 in tumorigenesis. ERKs are a family of protein-serine/threonine kinases, which serve vital roles in the control of diverse cell functions, such as cell differentiation, proliferation and survival, by phosphorylating several substrates including transcription factors, protein kinases and phosphatases (30). Increasing evidence has suggested that amplification or activation of ERK signaling frequently occurs in several malignant tumors, such as gastric adenocarcinoma and lung cancer $(31,32)$, which results in increased cell proliferation, promotion of cell cycle progression and repressed apoptosis of tumor cells (33). Given that the ERK signaling pathway participates in several aspects of tumorigenesis by regulating the expression of its downstream signaling molecules, such as NF- $\kappa \mathrm{B}$ (34), Bcl-2 (35) and STAT3 (36), the present study assessed whether the expression levels of these genes were affected following USP18-knockdown. The results demonstrated that Bcl-2 and STAT3 expression was upregulated, whereas $\mathrm{NF}-\kappa \mathrm{B}$ expression remained unchanged (data not shown) following USP18-knockdown in HeLa cells. Bcl-2 is a novel gene encoding a unique apoptosis inhibitor that efficiently suppresses apoptosis induced by the p53 tumor suppressor protein (37). STAT3 is an important proto-oncogene essential for modulating the transition from the $G_{1}$ to $S$ phase of the cell cycle (38), and most cancer cases arise due to proliferating cells losing control of cell cycle regulation, in which loss of the $\mathrm{G}_{1} / \mathrm{S}$-phase transition checkpoint is a major cause of cancer (39).

In conclusion, the results of the presents study demonstrated that USP18 expression was downregulated in UCC tissues, and USP18-knockdown facilitated tumor cell proliferation and migration by affecting the expression levels of genes associated with the ERK signaling pathway. Overall, the current results provide a novel mechanism for USP18-deficiency, which may serve a crucial role in UCC progression in an ERK-dependent manner.

\section{Acknowledgements}

Not applicable.

\section{Funding}

The present study was supported by the National Natural Science Foundation of China (grant no. 81272854), Key 
Projects of Natural Science Foundation of Heilongjiang Province (grant no. ZD2019H008), Excellent Innovation Team Construction Project of Basic Scientific Research Business Fee of Provincial Colleges and Universities in Heilongjiang Province (grant no. 2019-KYYWF-1334), Double First-class Discipline Construction Project in Heilongjiang Province (grant name. northern medicine and functional food), Young innovative talents training project of regular undergraduate colleges and universities in Heilongjiang Province (grant no. UNPYSCT-2020054), Youth Academic Backbone Support Program for Institution of Common Higher Education in Heilongjiang Province (grant no. 1252G059), Personnel Training Project of Basic Scientific Research Business Expenses of Department of Education in Heilongjiang Province (grant no. 2019-KYYWF-1338), Science and Innovation Team Foundation of Jiamusi University (grant no. cxtd-2016-03) and Biology Team Project of Jiamusi University (grant no. jdxktd-2019003).

\section{Availability of data and materials}

The datasets used and/or analyzed during the current study are available from the corresponding author on reasonable request.

\section{Authors' contributions}

AP and YL participated in cell experiments and drafted the manuscript. JG and PZ participated in statistical analyses. YH, LS and JRW performed immunohistochemistry assay. $\mathrm{CZ}, \mathrm{YC}$, and QR participated in cell transfection and cell experiments. SL, SF and TZ performed western blot analyses. AP, YL and JTW confirm the authenticity of all the raw data. WW and JTW designed the study and performed the revision of the manuscript. All authors read and approved the final manuscript.

\section{Ethics approval and consent to participate}

The medical ethics committee at Jiamusi University (Jiamusi, China) approved all procedures performed in the present study involving animals and human participants, which were in accordance with ethical standards, and all patients provided written informed consent prior to participation in this study.

\section{Patient consent for publication}

Not applicable.

\section{Competing interests}

The authors declare that they have no competing interests.

\section{References}

1. Van Hede D, Langers I, Delvenne P and Jacobs N: Origin and immunoescape of uterine cervical cancer. Presse Med 43: e413-e421, 2014

2. Bray F, Ferlay J, Colombet M, Soerjomataram I, Siegel RL, Torre A and Jemal A: Global cancer statistics 2018: GLOBOCAN estimates of incidence and mortality worldwide for 36 cancers in 185 countries. CA Cancer J Clin 68: 394-424, 2018.
3. Arbyn M, Weiderpass E, Bruni L, de Sanjosé S, Saraiya M, Ferlay J and Bray F: Estimates of incidence and mortality of cervical cancer in 2018: A worldwide analysis. Lancet Glob Health 8: e191-e203, 2020.

4. Oikonomaki M, Bady P and Hegi ME: Ubiquitin specific peptidase 15 (USP15) suppresses glioblastoma cell growth via stabilization of HECTD1 E3 ligase attenuating WNT pathway activity. Oncotarget 8: 110490-110502, 2017.

5. Baker RT, Wang XW, Woollatt E, White JA and Sutherland GR: Identification, functional characterization, and chromosomal localization of USP15, a novel human ubiquitin-specific protease related to the UNP oncoprotein, and a systematic nomenclature for human ubiquitin-specific proteases. Genomics 59: 264-274, 1999.

6. Wilkinson KD: Regulation of ubiquitin-dependent processes by deubiquitinating enzymes. FASEBJ 11: 1245-1256, 1997.

7. Chung $\mathrm{CH}$ and Baek SH: Deubiquitinating enzymes: Their diversity and emerging roles. Biochem Biophys Res Commun 266: 633-640, 1999.

8. Liu LQ, Ilaria R Jr, Kingsley PD, Iwama A, van Etten RA, Palis J and Zhang DE: A novel ubiquitin-specific protease, UBP43, cloned from leukemia fusion protein AML1-ETO-expressing mice, functions in hematopoietic cell differentiation. Mol Cell Biol 19: 3029-3038, 1999.

9. Malakhov MP, Malakhova OA, Kim KI, Ritchie KJ and Zhang DE: UBP43 (USP18) specifically removes ISG 15 from conjugated proteins. J Biol Chem 277: 9976-9981, 2002.

10. Honke N, Shaabani N, Zhang DE, Hardt C and Lang KS: Multiple functions of USP18. Cell Death Dis 7: e2444, 2016.

11. Friedrich SK, Schmitz R, Bergerhausen M, Lang J, Cham LB, Duhan V, Häussinger D, Hardt C, Addo M, Prinz M, et al: Usp18 expression in $\mathrm{CD} 169^{+}$macrophages is important for strong immune response after vaccination with VSV-EBOV. Vaccines (Basel) 8: 142, 2020.

12. Liu X, Li H, Zhong B, Blonska M, Gorjestani S, Yan M, Tian Q, Zhang DE, Lin X and Dong C: USP18 inhibits NF- $\kappa$ B and NFAT activation during Th17 differentiation by deubiquitinating the TAK1-TAB1 complex. J Exp Med 210: 1575-1590, 2013.

13. Dziamałek-Macioszczyk P, Haraźna J and Stompór T: Versatility of USP18 in physiology and pathophysiology. Acta Biochim Pol 66: 389-392, 2019.

14. Cai X, Feng S, Zhang J, Qiu W, Qian M and Wang Y: USP18 deubiquitinates and stabilizes Twist1 to promote epithelial-mesenchymal transition in glioblastoma cells. Am J Cancer Res 10: 1156-1169, 2020.

15. Tong HV, Hoan NX, Binh MT, Quyen DT, Meyer CG, Hang DT, Hang DT, Son HA, Van Luong H, Thuan ND, et al: Upregulation of Enzymes involved in ISGylation and Ubiquitination in patients with hepatocellular carcinoma. Int J Med Sci 17: 347-353, 2020.

16. Kim YH, Kim WT, Jeong P, Ha YS, Kang HW, Yun SJ, Moon SK, Choi YH, Kim IY and Kim WJ: Novel combination markers for predicting survival in patients with muscle invasive bladder cancer: USP18 and DGCR2. J Korean Med Sci 29: 351-356, 2014.

17. Tan Y, Zhou G, Wang X, Chen W and Gao H: USP18 promotes breast cancer growth by upregulating EGFR and activating the AKT/Skp2 pathway. Int J Oncol 53: 371-383, 2018.

18. Burkart C, Arimoto K, Tang T, Cong X, Xiao N, Liu YC, Kotenko SV, Ellies LG and Zhang DE: Usp18 deficient mammary epithelial cells create an antitumour environment driven by hypersensitivity to IFN- $\lambda$ and elevated secretion of $\mathrm{Cxcl} 10$. EMBO Mol Med 5: 1035-1050, 2013.

19. Potu H, Sgorbissa A and Brancolini C: Identification of USP18 as an important regulator of the susceptibility to IFN-alpha and drug-induced apoptosis. Cancer Res 70: 655-665, 2010.

20. Hong B, Li H, Lu Y, Zhang M, Zheng Y, Qian J and Yi Q: USP18 is crucial for IFN- $\gamma$-mediated inhibition of B16 melanoma tumorigenesis and antitumor immunity. Mol Cancer 13: 132, 2014.

21. Wu S, Shang H, Cui L, Zhang Z, Zhang Y, Li Y, Wu J, Li RK and Xie J: Targeted blockade of interleukin-8 abrogates its promotion of cervical cancer growth and metastasis. Mol Cell Biochem 375: 69-79, 2013.

22. François-Newton V, Magno de Freitas Almeida G, PayelleBrogard B, Monneron D, Pichard-Garcia L, Piehler J, Pellegrini S and Uzé G: USP18-Based negative feedback control is induced by type I and type III interferons and specifically inactivates interferon a response. PLoS One 6: e22200, 2011.

23. Fan W, Xie S, Zhao X, Li N, Chang C, Li L, Yu G, Chi X, Pan Y, Niu J, et al: IFN- $\lambda 4$ desensitizes the response to IFN- $\alpha$ treatment in chronic hepatitis $\mathrm{C}$ through long-term induction of USP18. J Gen Virol 97: 2210-2220, 2016. 
24. Dagenais-Lussier X, Loucif $\mathrm{H}$, Cadorel H, Blumberger J, Isnard S, Bego MG, Cohen ÉA, Routy JP and van Grevenynghe J; Montreal Primary Infection Study Group: USP18 is a significant driver of memory CD4 T-cell reduced viability caused by type I IFN signaling during primary HIV-1 infection. PLoS Pathog 15: e1008060, 2019.

25. Kang JA and Jeon YJ: Emerging roles of USP18: From biology to pathophysiology. Int J Mol Sci 21: 6825, 2020.

26. Chinyengetere F, Sekula DJ, Lu Y, Giustini AJ, Sanglikar A Kawakami M, Ma T, Burkett SS, Eisenberg BL, Wells WA, et al: Mice null for the deubiquitinase USP18 spontaneously develop leiomyosarcomas. BMC Cancer 15: 886, 2015.

27. Diao W, Guo Q, Zhu C, Song Y, Feng H, Cao Y, Du M and Chen H: USP18 promotes cell proliferation and suppressed apoptosis in cervical cancer cells via activating AKT signaling pathway. BMC Cancer 20: 741, 2020

28. Mustachio LM, Kawakami M, Lu Y, Rodriguez-Canales J, Mino B, Behrens C, Wistuba I, Bota-Rabassedas N, Yu J, Lee JJ, et al: The ISG15-specific protease USP18 regulates stability of PTEN. Oncotarget 8: 3-14, 2017.

29. Gu T, Lu L, An C, Zhang Y, Wu X, Xu Q and Chen G: Negative regulation of the RLR-mediated IFN signaling pathway by duck ubiquitin-specific protease 18 (USP18). J Cell Physiol 234: 3995-4004, 2019.

30. Yoon $\mathrm{S}$ and Seger R: The extracellular signal-regulated kinase: Multiple substrates regulate diverse cellular functions. Growth Factors 24: 21-44, 2006.

31. Bang YJ, Kwon JH, Kang SH, Kim JW and Yang YC: Increased MAPK activity and MKP-1 overexpression in human gastric adenocarcinoma. Biochem Biophys Res Commun 250: 43-47, 1998.

32. Tang Q, Wu J, Zheng F, Hann SS and Chen Y: Emodin increases expression of insulin-like growth factor binding protein 1 through activation of MEK/ERK/AMPK $\alpha$ and interaction of PPAR $\gamma$ and Sp1 in lung cancer. Cell Physiol Biochem 41: 339-357, 2017.
33. Guo YJ, Pan WW, Liu SB, Shen ZF, Xu Y and Hu LL: ERK/MAPK signalling pathway and tumorigenesis. Exp Ther Med 193: 1997-2007, 2020

34. Lin CW, Shen SC, Chien CC, Yang LY, Shia LT and Chen YC: 12-O-tetradecanoylphorbol-13-acetate-induced invasion/migration of glioblastoma cells through activating PKCalpha/ERK/NF-kappaB-dependent MMP-9 expression. J Cell Physiol 225: 472-481, 2010.

35. Yang T, Xu F, Sheng Y, Zhang W and Chen Y: A targeted proteomics approach to the quantitative analysis of ERK/Bcl-2-mediated anti-apoptosis and multi-drug resistance in breast cancer. Anal Bioanal Chem 408: 7491-7503, 2016.

36. Mu X, Shi W, Xu Y, Xu C, Zhao T, Geng B, Yang J, Pan J, Hu S, Zhang C, et al: Tumor-derived lactate induces M2 macrophage polarization via the activation of the ERK/STAT3 signaling pathway in breast cancer. Cell Cycle 17: 428-438, 2018.

37. Miyake H, Hanada N, Nakamura H, Kagawa S, Fujiwara T, Hara I, Eto H, Gohji K, Arakawa S, Kamidono S and Saya H: Overexpression of Bcl-2 in bladder cancer cells inhibits apoptosis induced by cisplatin and adenoviral-mediated p 53 gene transfer. Oncogene 16: 933-943, 1998.

38. Luo J, Yan R, He X and He J: Constitutive activation of STAT3 and cyclin D1 overexpression contribute to proliferation, migration and invasion in gastric cancer cells. Am J Transl Res 9: 5671-5677, 2107.

39. Ragkousi K and Gibson MC: Epithelial integrity and cell division: Concerted cell cycle control. Cell Cycle 17: 399-400, 2018.

(i) (5) $\odot$ This work is licensed under a Creative Commons Attribution-NonCommercial-NoDerivatives 4.0 International (CC BY-NC-ND 4.0) License. 